\title{
Curcumin Nanoparticles and Blue Laser Irradiation in Photothermal Inactivation of Selected Oral Pathogens in Vitro
}

\author{
Pavel Polenik \\ Charles University, Medical Faculty \\ Alej Svobody 80, Czech Republic \\ polenik@ fnplzen.cz
}

\begin{abstract}
In last years, devices emitting laser beam in blue spectrum has been the subject of substantial research also in dentistry. But improvement of bactericidal effect might be theoretically possible using exogenous chromophore under the terms of photothermal therapy. With respect to the color, curcumin seems be a suitable chromophore for this method and we decided to use curcumin nanoparticles and evaluate their effect as a photosensitizer for $445 \mathrm{~nm}$ laser in effectiveness against selected $G^{+}$and $G^{-}$bacteria in vitro. Curcumin nanoparticles were prepared using ultrasound assisted solvent-antisolvent precipitation. Diode laser $445 \mathrm{~nm}$ (SIROLaser Blue) was used as a source of laser irradiation $(100 \mathrm{~mW}, 1 \mathrm{~min}$. exposition time, energy density $\left.7,68 \mathrm{~J} / \mathrm{cm}^{2}\right)$. Bacterial strains of Parvimonas micra and Enterococcus faecalis were cultivated on solid media in marked areas covered by curcumin nanoparticles without and with subsequent sonication. After laser irradiation and anaerobic cultivation, the bacterial growth was evaluated. Irradiated areas without contact with curcumin nanoparticles or nanoparticles without sonication showed intact bacterial colonies. Completely different quality was detectable in cases of irradiated colonies growing in contact with sonicated curcumin nanoparticles. Destruction of bacterial colonies was clearly visible and repeated cultivation of taken material was without positive response confirming presence of non-living cells inside of colonies. Results of our preliminary study showed promising direction of laser photothermal therapy using curcumin nanoparticles in reduction or elimination of oral microflora especially in locations with poor access for classic therapeutic effect.
\end{abstract}

Keywords: photothermal effect, curcumin, nanoparticles, blue laser.

(C) Copyright 2020 Authors - This is an Open Access article published under the Creative Commons Attribution License terms (http://creativecommons.org/licenses/by/3.0). Unrestricted use, distribution, and reproduction in any medium are permitted, provided the original work is properly cited.

\section{Introduction}

More than 750 types of bacteria or phylotypes are detected in oral cavity. Despite the fact, that human immune system constantly monitores their growth and reproduction, some of the bacteria in the mouth are responsible for oral diseases such as caries and periodontal diseases, which are one of the most common diseases in humans. Prevention and treatment are focused consequently on reduction of bacterial load of oral structures. According to the location of bacterial biofilm, different methods of antibacterial strategy can be used. Serious problem is accessibility of bacterial communities localized in deep periodontal pockets and, also between and inside of epithelial cells. Systemic application of antibiotics is frequently the method of choice in these cases. But the undesirable side effect of such therapeutic access is general decrease of sensitivity to antibiotics. So that the continual looking for an alternative method is the imperative of present time.

Light from high-power lasers is known to be bactericidal and investigations have shown that it is effective against organisms implicated in caries and inflammatory periodontal diseases. Although light from low-power lasers has no adverse effect on bacterial viability, bacteria can be sensitized to killing by such light by prior treatment with a chemical 
photosensitizing agent. The advantages of the technique are that killing is achieved in very short periods of time (<60 s), resistance development in the target bacteria would be unlikely and damage to adjacent host tissues can be avoided. This approach may be a useful alternative to antibiotics and antiseptics in eliminating cariogenic and periodontopathogenic bacteria from disease lesions.

Moreover, laser delivery systems have more better access to the locations where the effectivity of classic mechanical methods of biofilm removal is questionable. Different wavelengths in combinations with responsible photosensitizers have been tested in connection with photodynamic (PDT) inactivation of selected oral pathogens. As PDT is an oxygen-dependent process, its use in deep periodontal pockets and infected root canals may be limited. From this point of view, killing of microorganisms through a local hyperthermal mechanism - antimicrobial photothermal therapy (PTT) might by more effective.

In last years, devices emitting laser beam in blue spectrum has been the subject of substantial research also in dentistry [1]. Previous investigations demonstrated phototoxic effect of blue light for effectively reducing some pathogens. But improvement of bactericidal effect might be theoretically possible using exogenous chromophore under the terms of PTT $[2,3]$. With respect to the color, curcumin seems be a suitable chromophore for this method. In addition to this activity, anti-inflammatory, antioxidant, chemopreventive and chemotherapeutic abilities may be beneficial in suppression of inflammatory reactions.

Important criterion of effectivity of photothermal reaction is accessibility and contact of bacteria with photosensitizer. Because curcumin shows poor solubility in water, we decided to use curcumin nanoparticles and evaluate their effect as a photosensitizer for $445 \mathrm{~nm}$ laser in effectiveness against selected $\mathrm{G}^{+}$and $\mathrm{G}^{-}$bacteria in vitro.

\section{Materials and methods}

\subsection{Curcumin nanoparticles preparation}

Ultrasonically-assisted liquid antisolvent precipitation was used for nanoparticles preparation. Stock of curcumin solution $(5 \mathrm{mg} / \mathrm{ml})$ was prepared by dissolving curcumin powder (Sigma Aldrich) in 90\% ethanol (sample E) or acetone (sample A) (20 ml). One $\mathrm{ml}$ of stock solution was added to boiling water $(50 \mathrm{ml})$ in drop-wise manner under ultrasonication condition with frequency of $50 \mathrm{kHz}$. The solution was sonicated for $30 \mathrm{~min}$. After sonication, the mixture was stirred at $800 \mathrm{rpm}$ for $20 \mathrm{~min}$ till the orange colored precipitate was obtained. Thereafter, supernatant was discarded and the pellet obtained was used for further study. Before application on the surface of solid cultivation media $100 \mathrm{mg}$ of curcumin nanomaterial was dissolved in $1 \mathrm{ml}$ of $0,9 \%$ saline solution. Paralelly, nanoparticles without sonication were prepared as well.

\subsection{Laser equipment}

Diode laser emitting $445 \mathrm{~nm}$ laser beam (SIROLaser Blue, Dentsply Sirona, Germany) was used for evaluation of spontaneous antibacterial laser effect and for induction of photothermal reaction in cooperation with curcumin nanoparticles.

\subsection{Bacterial cultures}

Bacterial strains of Parvimonas micra ATCC 33270 and Enterococcus faecalis ATCC 29212 were obtained from Thermo-Scientific (Lenexa, KS, USA). Both strains were cultivated into anaerobe basal broth (Oxoid, Basingstoke, Hampshire, England) under anaerobic conditions for 48 hours at $37^{\circ} \mathrm{C}$. Then $P$. micra was inoculated on anaerobe basal agar (Oxoid, Basingstoke, Hampshire, England) supplemented with $5 \%$ sterile defibrinated horse blood. Two areas on the left side of $P$. micra inoculum were covered one by one drop of curcumin nanoparticles prepared using ethanol or acetone, two areas on the right side by curcumin sonicated nanoparticles. Subsequent cultivation taken place under anaerobic conditions to the mid-log phase. Homogenous growth of bacterial colonies including areas covered by different types of nanoparticles was visible after 72 hours of cultivation [Figure 1]. 


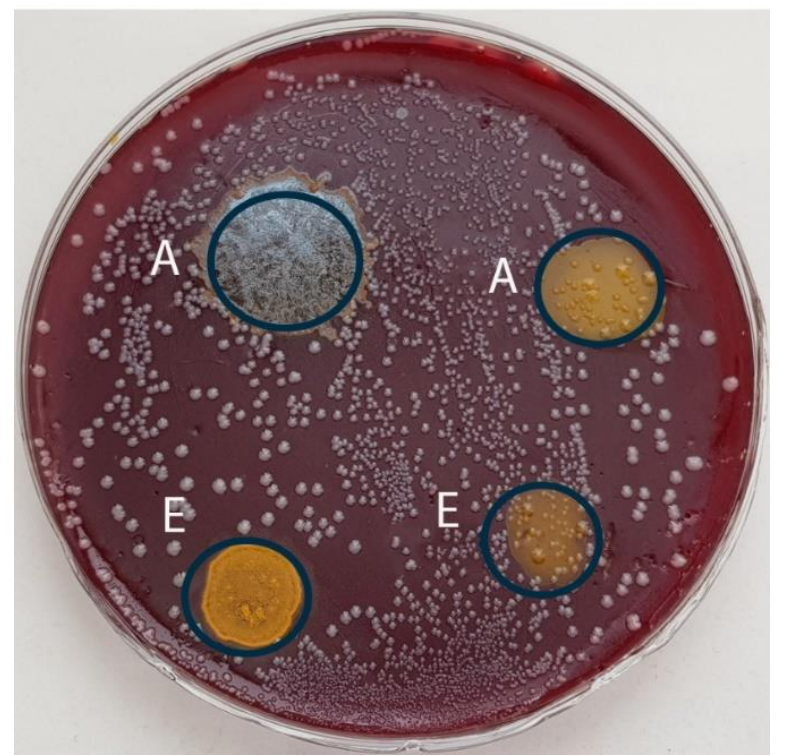

Figure1. Colonies of $P$. micra covering curcumin nanoparticles. A- solvent $=$ acetone, $\mathrm{E}-$ solvent $=$ ethanol .

E. faecalis was inoculated on Slanetz\&Bartley medium (Oxoid, Basingstoke, Hampshire, England). Two areas on the left side were covered one by one drop of curcumin nanoparticles in two used solvents (ethanol, acetone) and two areas in the right side by curcumin sonicated nanoparticles. After 72 hours of anaerobic cultivation at $37^{\circ} \mathrm{C}$ the bacterial growth was evaluated.

All marked areas of $P$. micra colonies covered by curcumin nanoparticles and one colonized area free of nanoparticles of the same extent were irradiated by diode laser. Following settings were used: $100 \mathrm{~mW}$ power, irradiation time $1 \mathrm{~min}$, fiber diameter $0,3 \mathrm{~mm}$, distance of fiber from the surface $15 \mathrm{~mm}$, energy density

$7,68 \mathrm{~J} / \mathrm{cm}^{2}$. After 48 hours of anaerobic cultivation at $37^{\circ} \mathrm{C}$ the antibacterial effect was evaluated.

The same process of curcumin nanoparticles activation was used in the group of $E$. faecalis experiments.

Complete test for each of observed bacteria was 10 times repeated.

\section{Results}

The same uniform picture was visible on the surfaces of tested bacterial cultures. In the group of P. micra, irradiated areas covered by curcumin nanoparticles without sonication showed intact bacterial colonies. Subsequent cultivation of samples from these colonies validated viability of colony forming bacteria. The same effect was visible after laser irradiation in the regions without nanoparticles application. Completely different quality was detectable in cases of irradiated colonies growing in contact with sonicated curcumin nanoparticles. Destruction of bacterial colonies was clearly visible and repeated cultivation of taken material didn't show positive response confirming presence of non-living cells inside colonies. The same bactericidal effect was observed in both used solvents (ethanol, acetone) [Figure 2].

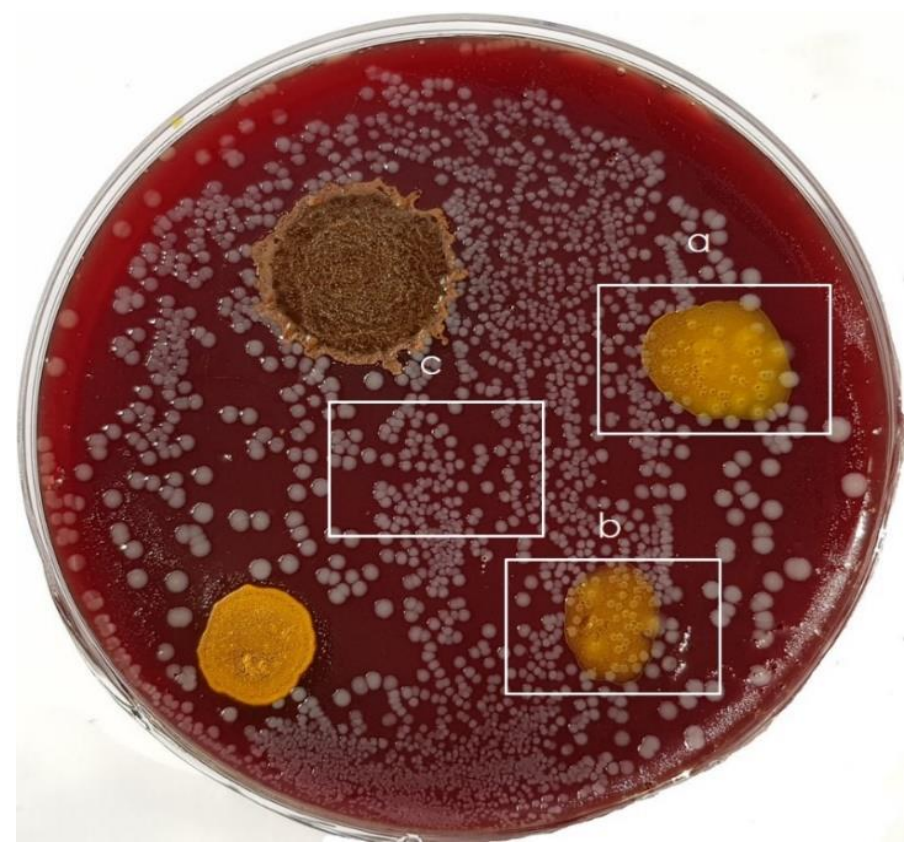

Figure 2. Colonies of P. micra after laser irradiation and subsequent cultivation. Areas $\mathbf{a}$ and $\mathbf{b}$ demonstrate disruptions of colonies growing on sonicated nanoparticles and area $\mathbf{c}$ intact colonies in absence of curcumin nanoparticles.

Interaction between nanoparticles prepared without sonication and E. faecalis cells located on the left side of cultivation dishes showed their intact homogenous growth. Taken cells samples from these areas demonstrated completely living cells during cultivation. For the effect of sonicated nanoparticles on the right half of cultivation dishes is typical destruction of bacterial cells and the effect is not dependent on the used solvent. 


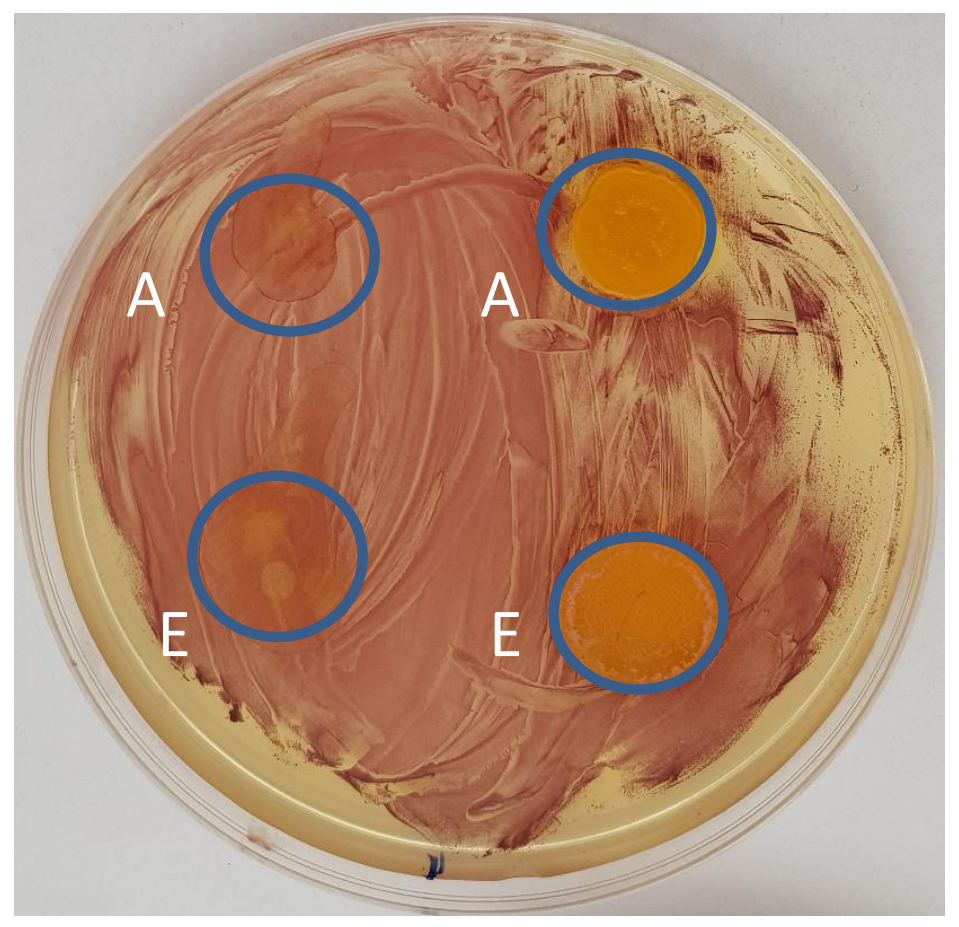

Figure 3. Bacterial culture of E. faecalis. Areas treated by curcumin nanoparticles without sonication after laser irradiation on the left side $=$ intact bacterial growth. Areas treated by sonicated nanoparticles after laser irradiation on the right side $=$ complete bacterial destruction. $\mathrm{A}-$ solvent $=$ acetone, $\mathrm{E}$ - solvent $=$ ethanol.

\section{Discussion}

The goal of antimicrobial therapy in dentistry is simple in principle yet often difficult to achieve: to eradicate periodontal pathogens in the oral cavity. The difficulty arises not from the antimicrobial agents themselves but from the complex array of reservoirs where these organisms can persist and means to effectively deliver antimicrobial agents to these locations. Important questions of antibacterial strategy are also different forms of bacterial colonization associated with dissimilar sensitivity to the antimicrobials. Especially in periodontology, the dominant problem is complexity of bacterial biofilm. It is possible to found in recent bibliography a rich list of substances with antibacterial effect, but the methods of evaluation are not usable for practical use. It is mostly detection of bactericidal activity in planktonic cultures, but in reality, the dominant problem is very resistant bacterial biofilm. In planktonic cultures curcumin solutions inhibited the growth of $P$. gingivalis in dosedependent manner and its MIC was $12 \mu \mathrm{g} / \mathrm{ml}$ [4]. On the other hand, concentration of $20 \mu \mathrm{g} / \mathrm{ml}$ was able to inhibit $P$. gingivalis biofilm formation by $>80 \%$ [5] and Bomdyal et al. studied that curcumin powder impeded the growth of $P$. gingivalis and $P$. intermedia only at higher concentration such as $100 \mu \mathrm{g} / \mathrm{ml}$ [6]. In a similar way, antibacterial activity of curcumin solution was evaluated against the standard strains of most predominant organisms of deep carious lesions namely Streptococcus mutans, Lactobacillus casei, A. actinomycetemcomitans, and most widespread strains of root canal bacteria, namely P. gingivalis, Prevotella intermedia, and Enterococcus faecalis. Their findings indicated the antibacterial activity of curcumin against all the investigated organisms apart from E. faecalis [7]. Except curcumin solution, also antibacterial effect of curcumin gel was tested in vitro or clinically. Positive inhibitory effect against $P$. gingivalis and $P$. intermedia was recorded in connection with $2 \%$ gel in an in vitro study [8]. Locally delivered $1 \%$ curcumin gel was more effective in inhibiting the growth of oral bacteria when used as an adjunct to scaling and root planing in the treatment of chronic periodontitis [9].

Nanotechnology strategies for curcumin delivery have been studied over the past years. A diverse array of novel preparations has been developed (nanocurcumins) and these nano-range formulations have been mostly limited to in vitro models of cancer. But some investigations have shown that the formulations have also potential for the treatment of infection diseases.

Next step in the use of curcumin is connected with laser application in the terms of photothermal therapy. In the context of combination of curcumin solutions and laser energy the reduction of salivary bacteria is better than curcumin or diode laser alone [10]. To a lesser extent was the research focused on evaluation of single-species [11] and multi-species biofilms [12]. On this field of activity is dominant problem accessibility of target structures and significant differences are typical for such antibacterial activities. 


\section{Conclusion}

Photothermal antimicrobial therapy is of great importance, particularly in an era when minimally invasive dentistry and prevention are at the forefront of the aims of dental medicine. In oral medicine, photothermal therapy has overcome problems associated with antibacterial, antiviral and antifungal resistance, and it can also be used against microbes as many times as needed.

Antibacterial effect of selected lasers is frequently based on endogenous pigments of some pathogens. Presented study showed limited ability of lasers to destroy non-pigmented bacterial cells. Curcumin nanoparticles developed using ultrasonically assisted liquid antisolvent precipitation after interaction with bacteria act as target chromophore for laser beam with subsequent photothermal reaction. Curcumin alone is not a mechanism of cell destruction and effective photothermal reaction was detected in connection with sonicated nanoparticles in contrast to nanoparticles without sonication. Sonication is useful to increase the active targeting of the photothermally-active particles in the colony-forming bacteria,

Very good accessibility of oral biotops for laser beam is a promising condition of oral antibacterial strategy based on interaction between laser and by curcumin nanoparticles labelled bacterial cells.

Even though promising for clinical application, this approach needs to be intensively investigated through preclinical studies and clinical trials.

\section{References}

[1] S. Shany.-Kdoshim, D. Polaka, Y. Houri-Haddadb, 0. Feuerstein, "Killing mechanism of bacteria within multi-species biofilm by blue light," J. Oral Microbiol., vol. 11, no.1, pp. 1-10, 2019

[2] L.I. Hyung-Jung, K. Si-Mook, J. Seung-Hwa, Ch.Ki-Ho, K. Baek-Il, " Antibacterial photodynamic therapy with curcumin and Curcuma xanthorrhiza extract against Streptococcus mutans," Photodiagnosis Photodyn. Ther., vol. 20, no. 4, pp. 116-119, 2017.

[3] M.R. Agel, E. Baghdan, S. R. Pinnapireddy, J. Lehmann, J. Schäfer, U. Bakowsky, " Curcumin loaded nanoparticles as efficient photoactive formulations against gram-positive and gram-negative bacteria,"
Colloids and Surfaces_B:_Biointerfaces, vol.178, no. 1, pp. 460-468, 2019.

[4] A. M. Sha, B. T. Garib, „Antibacterial effect of curcumin against clinically isolated Porphyromonas gingivalis and connective tissue reactions to curcumin gel in the subcutaneous tissue of rats," BioMed Res. Int.,vol.2019, article 6810936, 14 pages.

[5] S. Izui, S. Sekine, K. Maeda, M. Kubowina, A. Amano, A.Takada, H. Nagata, „Curcumin inhibited the growth of P. gingivalis in dose-dependent manner," $J$. Periodontol., vol. 87, no.1, pp. 83-90, 2016.

[6] R.S. Bomdyal, M.U. Shah, Y.S. Doshi, V.A. Shah, S.P. Khirade, „Antibacterial activity of curcumin (turmeric) against periopathogens: an in vitro evaluation," J. Adv. Clin. Res. Insights, vol.4, no. 6, pp.175-80, 2017.

[7] P.S. Mandroli, K. Bhat, „An in-vitro evaluation of antibacterial activity of curcumin against common endodontic bacteria," JAPS, vol.3, no. 10, pp.106-108, 2013.

[8] K. Rudhra, I. Paavai, Ch. Veejai, T. Abirami, A.V. Vummidi, A. Mahalingam, „An in vitro evaluation of curcumin oral gel antimicrobial efficacy against Porphyromonas gingivalis and Prevotella intermedia,"Drug Invention Today, vol 13, no 1, pp. 97-101, 2020.

[9] B. Madhu, S. S. Urolagin, K. B. Pentyala, S. B. Urolagin, M. S. Bhoi, „Novel Therapeutic Approach for the Treatment of Periodontitis by Curcumin," J. Clin. Diagn. Res., vol. 8, no. 12, pp. 65-69, 2014.

[10] D. P. Leite, F. R., Paolillo, T. N. Parmesano, C. R. Fontana, V. S. Bagnato, „Photomed. Laser Surg., vol. 32, no. 11, pp. 627-632, 2014.

[11] M. C. Andrade, A. P. D. Ribeiro, L. N. Dovigo, I. L. Brunetti, E. T., Giampaolo, „Effect of different preirradiation times on curcumin mediated photodynamic therapy against planktonic cultures and biofilm of Candida spp.," Arch. Oral Biol., vol. 58, no. 2, pp. 200-210, 2013.

[12] N. C. Zaranjo, C. R. Fontana, V. S. Bagnato, M. E. M. Gerbi, „, Photodynamic antibacterial therapy of curcumin in biofilms and carious dentine," Lasers Med. Sci., vol. 29, no. 2., pp. 629-635, 2014. 